\section{A retrospective analysis of bronchoscope guided and non- bronchoscope guided bronchoalveolar lavage for diagnosing lung disease in dogs}

\section{Samantha Evers, Erica Tinson}

Royal Veterinary College, Hatfield, United Kingdom

\section{OBJECTIVES}

Bronchoalveolar lavage (BAL) is a useful technique in diagnosing lung disease and can be performed with or without bronchoscopic guidance. A retrospective analysis was performed to compare the diagnostic capabilities of bronchoscope guided and non-bronchoscope guided BAL.

\section{METHODS}

Cytology and culture records from guided and nonguided BAL performed on 231 dogs presenting to a referral hospital were analyzed to determine how well the samples fit with the clinical diagnosis. Cytology reports were classified as 'definitive', 'supportive', or 'not helpful' based on the expected cytological profile of the diagnosis. Sensitivity was defined as the probability of a positive culture in a patient with a lower respiratory tract infection, while specificity was defined as the probability of a negative culture in a patient with a non-infectious diagnosis.

\section{RESULTS}

Bronchoscope guided BAL (B-BAL) was definitive or supportive in 102/116 (88\%) cases while non-bronchoscope guided BAL (NB-BAL) was definitive or supportive in $115 / 126(91 \%)$ cases. The sensitivity and specificity were found to be $67 \%$ and $91 \%$ respectively for $\mathrm{B}-\mathrm{BAL}$ and $72 \%$ and $89 \%$ respectively for NB-BAL. No significant differences were found between methods in diagnosing infectious, parasitic, neoplastic, and chronic lung diseases or in sensitivity and specificity of culture results.

\section{STATEMENT (CONCLUSIONS)}

This study provides new evidence that B-BAL and NB$B A L$ could be equally effective. As NB-BAL is faster, less expensive, and safer for the patient, these results could have significant implications for first opinion, emergency, and referral hospitals alike. However, further prospective studies are needed to confirm these findings.

\section{Intranasal} meningoencephalocele in a one-year-old Staffordshire Bull Terrier with unilateral destructive rhinitis

\section{Sofia Rapastella, Robert Brash, Heather Covey, Mellora Sharman}

Animal Health Trust, Kentford, Newmarket, United Kingdom

\section{OBJECTIVES}

The aim is to describe a case of unilateral destructive rhinitis associated with intranasal meningoencephalocele in a young dog with chronic rhinorrhoea and epistaxis.

\section{METHODS}

A 18-month-old male neutered Staffordshire Bull Terrier presented for investigations of chronic sneezing, left-sided rhinorrhoea and epistaxis associated with nasal planum depigmentation and pruritus. Investigations included physical examination, neurological assessment, haematology, biochemistry, computed tomography of head and thorax, rhinoscopy. Endoscopic biopsies were collected for histology, bacterial and fungal culture.

\section{RESULTS}

Physical examination showed reduced airflow through the left nostril without bone asymmetry or depigmentation. Computed tomography revealed severe destruction of the left nasal conchae and complete absence of the cribriform plate, with protrusion of the left olfactory lobe into the ethmoidal nasal cavity. There was no evidence of meningitis. Rhinoscopy did not identify fungal plaques or foreign material. Nasal biopsies revealed lymphoplasmacytic destructive rhinitis. Bacterial and fungal cultures were negative. The clinical signs completely resolved after two weeks doxycycline treatment.

Congenital meningoencephalocele is reported to cause cerebrospinal fluid rhinorrhoea and secondary destructive rhinitis in humans, which could also explain the findings in this case. However, unilateral destructive rhinitis could cause cribriform plate lysis and secondary meningoencephalocele, although this is considered less likely here since the cribriform erosion was disproportionate compared to the cranial bones. 